\title{
Private online channels and student-centred interaction
}

\section{James McMenamin}

University of Applied Sciences and Arts, Switzerland

\section{Marie-Thérèse Rudolf von Rohr}

University of Applied Sciences and Arts, Switzerland

Keywords: online learning; student-centred interaction; emergency remote teaching; learning communities; Covid-19.

\section{The challenge}

In 2020, online teaching became mandatory at our institution, the University of Applied Sciences and Arts (UAS), Northwestern Switzerland, due to the Covid-19 pandemic. As lecturers of English for Professional and Academic Purposes at the School of Engineering, we faced the challenge of adapting our heavily interaction- and group-work based language courses for an emergency remote teaching setting (Hodges et al., 2020).

As regards technological measures, the UAS chose to supplement our traditional learning management system (LMS), Moodle, with web-conferencing software, WebEx and Zoom. However, despite our initial relief to still be able to teach, some drawbacks associated with the use of these conferencing tools quickly surfaced. Spoken or written interactions were lost when WebEx or Zoom sessions were closed or the internet connection was lost. Furthermore, due to the technical affordances of WebEx and Zoom, our sessions tended to be teacher-centred in the sense that they were always started by teachers, and it appeared that no spontaneous student-student interactions took place. In fact, as far as we could see, whether student-student interactions were to occur was entirely dependent on what group work teachers had planned for a particular lesson. Ironically, we found the use of electronic media for online content delivery, instead of making teaching and learning more individualised and learner-centred, brought us back to a challenge we had faced before Covid-19, namely the difficulty of facilitating meaningful student-student interactions inside and outside of class time. Interaction is key to learning, even more so 
for second language acquisition (Tran, 2018), and a sense of community needs to be fostered to increase the effectiveness of any course (Darby and Lang, 2019; Lave and Wenger, 2019).

\section{The response}

Through a survey conducted by the student council at the School, it came to our attention that students enjoyed using the collaborative platform Microsoft Teams. To accommodate students' preferences, we decided therefore to rely entirely on Teams to organise and host online courses in the Autumn semester of 2020. In our view, Teams offered an interface which had an advantage over other solutions, in that 'a team' resembled a virtual room where students could enter at any time and either chat or set up an online meeting. In addition, the chat rooms of online meetings in Teams would automatically be retained after class had finished, unlike with Zoom or WebEx. We also set up customised channels for group work within a team, in which students could hold independent meetings, which we had not previously been able to do.

As was perhaps to be expected, our use of Teams did not lead to an overnight revolution in our teaching. Class meetings were still initiated and closed by us. However, we gradually discovered that Teams offers some unexpected advantages over WebEx and Zoom. Below is a brief and non-exhaustive list of developments that we view as relevant to student-student interaction:

- Drag-and-drop file sharing and chat functionality make for uncomplicated sharing of information synchronously and asynchronously.

- Students seem to enjoy using emojis and GIFs to quickly comment on other students' posts, encouraging further interaction and keeping the mood light.

- In particular, the use of private channels (online rooms available only to certain individuals) enabled students to share data within groups, offering an independent online location for personal interactions during class and asynchronously. For example, in one case, a pair of students met outside class to practise for an upcoming oral examination. In another context, students used the chat function of their private channels to compare notes and exchange solutions during a reading comprehension task. 
- Once the software has been set up and started, no further login procedure was required.

- Any Word document uploaded to the Files section of Teams can, in the manner of a Google doc, be edited by all group members, making it easy to set up collaborative tasks.

\section{Recommendations}

In our view, student-student interaction can be facilitated in various ways and is not necessarily linked to the use of a single tool. However, the need for student-student interaction should be considered a crucial factor when planning online classes (Means, Murphy and Bakia, 2014; Darby and Lang, 2019). Our key insights here are:

a) Students will form online communities and groups to solve problems jointly if they are assigned collaborative tasks and have their own virtual room (private channel) where they can meet independently.

b) Group chats and documents which students can collaboratively edit can increase interactivity during online classes, and the option of referring to them after class benefits both teachers and students.

c) Students find it easier to use one collaborative platform than a combination of LMS and conferencing tools.

Despite our generally positive experiences working with Teams, it is not a panacea, and it is not the intention of this short paper to present it as such. For one thing, Teams cannot replace all the functionalities of Moodle, which can be used to specifically plan, enhance, and implement the learning process. Moreover, Moodle is open-source and as such, does not share student data with a large US corporation, as might be the case with Teams. This criticism cannot be taken lightly, yet we felt that the advantages outweighed said concerns in a time of crisis.

At present, the use of Teams is limited to a small handful of teachers at our institution. Because we are using it 'at our own risk' in the sense that it is not supported by our IT department, it is unlikely that many teaching colleagues will abandon WebEx or other supported web conferencing solutions. We would argue that features such as private 
channels and chat functionality tend to foster student-centred interaction, which is key to learning (Darby and Lang, 2019; Lave and Wenger, 2019). Therefore, such features should be more widely implemented in conferencing tools, and not limited to proprietary software such as Teams.

\section{References}

Darby, F., and Lang, J. M. (2019) Small teaching online applying learning science in online classes. San Francisco: Wiley Brand.

Hodges, C., Moore, S., Lockee, B., Trust, T., and Bond, A. (2020) The difference between emergency remote teaching and online learning. Available at:

https://er.educause.edu/articles/2020/3/the-difference-between-emergency-remoteteaching-and-online-learning (Accessed: 4 May 2021).

Lave, J., and Wenger, E. (2019) Situated learning: legitimate peripheral participation (30th printing). Cambridge: Cambridge University Press.

Means, B., Murphy, R., and Bakia, M. (2014) Learning online what research tells us about whether, when and how. New York: Routledge.

Tran, H. Q. (2018) 'Language alternation during L2 classroom discussion tasks', in Filipi, A. and Markee, N. (eds.) Conversation analysis and language alternation. Amsterdam: John Benjamins Publishing Company, pp.165-182.

\section{Author details}

James McMenamin is a lecturer of English for Specific Academic Purposes. His research interests include the use of technology to create online learning communities.

Marie-Thérèse Rudolf von Rohr is a lecturer of English for Specific Academic Purposes. Her fields of interests include interpersonal pragmatics, online learning, and studentcentred interaction. 